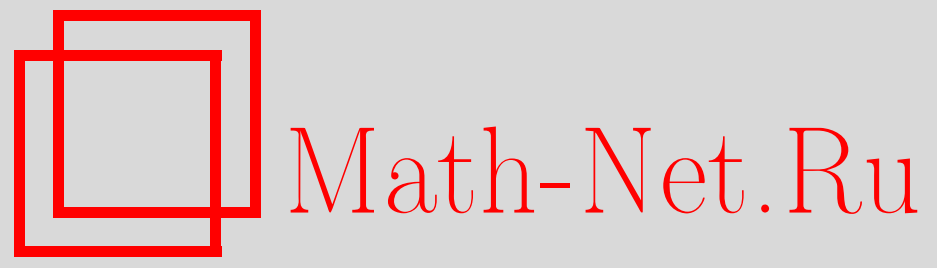

P. A. Zanzotto, Representation of a class of semimartingales as stable integrals, Теория вероятн. и ее примен., 1998, том 43, выпуск 4, 808-818

DOI: https://doi.org/10.4213/tvp2171

Использование Общероссийского математического портала MathNet.Ru подразумевает, что вы прочитали и согласны с пользовательским соглашением

http://www . mathnet.ru/rus/agreement

Параметры загрузки:

IP: 54.209 .52 .79

26 апреля 2023 г., 03:50:48

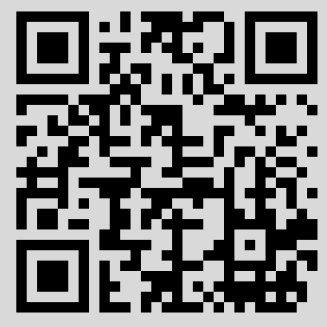


20. Kusuoka $S$. The nonlinear transformation of Gaussian measure on Banach space and its absolute continuity, I, II. - J. Fac. Sci. Univ. Tokyo, sec. 1A, 1982, v. 29, № 3, p. $567-598 ; 1983$, v. 30 , № 1 , p. 199-220.

21. Piech M. A. Differentiability of measures associated with parabolic equation on infinite dimensional spaces. - Trans. Amer. Math. Soc., 1979, v. 253, p. 191-209.

22. Pitcher T. S. Likelihood ratios for diffusion processes with shifted mean value. Trans. Amer. Math. Soc., 1961, v. 101, p. 168-176.

23. Shigekawa I. Existence of invariant measures of diffusions on an abstract Wiener space. - Osaka J. Math., 1987, v. 24, № 1, p. 37-59.

24. Simao I. Regular transition densities for infinite-dimensional diffusions. - Stochastic Anal. Appl., 1993, v. 11, № 3, p. 309-336.

25. Толмачев $H$. A. Об одном свойстве распределений диффузионных процессов. Матем. заметки, 1993, т. 54, № 3, с. 106-113.

26. Федерер Г. Геометрическая теория меры. М.: Наука, 1987.

27. Шевчик B. В. О подпространствах банахова пространства, совпадающих с областями значений линейных непрерывных операторов. - Докл. АН СССР, 1982, т. 263, c. $817-819$.

Поступила в редакцию

$1998 \mathrm{r}$.

ZANZOTTO P.A.*

\section{REPRESENTATION OF A CLASS OF SEMIMARTINGALES AS STABLE INTEGRALS}

Описывается класс семимартингалов, представимых в виде стохастического интеграла по $\alpha$-устойчивому процессу Леви. Условия сформулированы в терминах характеристик семимартингалов.

Ключевые слова и Фразы: семимартингалы без непрерывной мартингальной части, мера скачков, компенсатор, строго $\alpha$-устойчивые процессы Леви, устойчивые интегралы.

Introduction. There are several similarities between the Itô's theory for Brownian integrals and the Itô-type stochastic integration theory with respect to strictly $\alpha$-stable Lévy processes $(0<\alpha \leqslant 2)$ (these are also called $\alpha$-stable Lévy motions). First of all from the point of view of the integrability conditions: let us consider indeed the real-valued processes $X$ and $H$ such that $X$ is Lévy strictly $\alpha$-stable while $H$ is predictable. Then, for every $X$, the stochastic integral process $\int_{0}^{t} H d X$ exists if and only if the paths of $H$ lie locally in $L^{\alpha}(d x)$ a.s. Also the inner clock property holds for the $\alpha$-stable stochastic integrals, i.e., such integrals can be reduced to a standard form by a suitable time change. For example, if $H$ is positive or $X$ is symmetric, the integral process $\int_{0}^{t} H d X$ has the same distribution as $X$, when viewed on the time scale given by the process $\int_{0}^{t}\left|H_{s}\right|^{\alpha} d s$. More precisely, in these cases there exists a process $X^{\prime}$ having the same law as $X$ such that a.s. $\int_{0}^{t} H_{s} d X_{s}=X^{\prime} \circ \int_{0}^{t}\left|H_{s}\right|^{\alpha} d s$. (In other cases other representations hold, see [8].)

In the class of symmetric $\alpha$-stable Lévy processes, Brownian motion corresponds to the parameter $\alpha=2$, so the above properties can be viewed as extensions to such a class Italy.

*Dipartimento di Matematica, Università di Pisa, Via F. Buonarroti, 2, 56127 Pisa, 
of classical properties of Brownian integrals and were proved in the symmetric case in [12], while the general case was studied in [8], where a variety of time change representations was established for stable integrals.

But there is also another analogy between the two theories which seems not to have been noticed so far. This is the object of the present paper.

Recall that if a real continuous local martingale $X$ has predictable covariation process $\langle X, X\rangle$ absolutely continuous with respect to Lebesgue measure, it can be represented as a stochastic integral with respect to a Wiener process. More precisely, let $H$ denote a predictable process such that a.s.

$$
\langle X, X\rangle_{t}=\int_{0}^{t} H_{s}^{2} d s \text { for all } t .
$$

Then there exists some Wiener process $Z$ (possibly defined on an extension of the original probability space) such that

$$
X_{t}=\int_{0}^{t} H_{s} d Z_{s}, \quad t \geqslant 0 .
$$

Now, if we plunge this representation result in the class of stable Lévy processes, what can be said? In other words, what processes can be represented in the form (1) with the $Z$ process an $\alpha$-stable Lévy motion?

In order to briefly illustrate our results, let $X$ denote a semimartingale with vanishing continuous martingale part which we call a quadratic pure jump semimartingale (according to the terminology used in [11, p. 63]). Assume that the jump-measure of $X$ has the compensator

$$
\pi(d s, d x)=\left|H_{s}\right|^{\alpha} q_{\alpha}(d s, d x),
$$

where $0<\alpha<2, H$ is a predictable process and $q_{\alpha}$ is the compensator of the jumpmeasure of an $\alpha$-stable Lévy motion, i.e., $q_{\alpha}(d s, d x)=d s \otimes \nu(d x), \nu$ denotes the Lévy measure $\left(\nu(d x)=\left(c /|x|^{1+\alpha}\right) d x\right.$ in the symmetric case).

Assume that $X$ is a purely discontinuous local martingale (resp. is of pure jump type with a locally finite variation) and that condition (2) holds for it with $\alpha, 1<\alpha<2$ (resp. $0<\alpha<1$ ) and $\nu$ symmetric.

Then there is an $\alpha$-stable Lévy motion $Z$ (possibly defined on some extension of the original probability space) with Lévy measure $\nu$ such that

$$
X_{t}=\int_{] 0, t]} H_{s} d Z_{s}, \quad t \geqslant 0
$$

(see Theorem 2, c) below).

If condition (2) holds with a $\nu$ not necessarily symmetric, the other assumptions being in force, we have the representation formula

$$
X_{t}=\int_{] 0, t]}\left|H_{s}\right| d Z_{s}, \quad t \geqslant 0
$$

with $Z$ as above (see Theorem 2, a) and b)).

The simplest case with $\alpha=1$ is stated as follows.

Assume that $X$ is a semimartingale satisfying condition (2) with $\alpha=1$ and such that, for all $t$,

$$
X_{t}=\int_{j 0, t]} \int_{\{|x| \leqslant 1\}} x(\mu-\pi)(d s, d x)+\int_{\} 0, t]} \int_{\{|x|>1\}} x \mu(d s, d x) .
$$


Then we have both the representations

$$
X_{t}=\int_{] 0, t]} H_{s} d Z_{s}, \quad \text { and } \quad X_{t}=\int_{] 0, t]}\left|H_{s}\right| d Z_{s}^{*}, \quad t \geqslant 0
$$

where $Z$ and $Z^{*}$ both are Cauchy processes, defined in general on an extension of the original probability space (see the corollary below).

Still in the case $\alpha=1$, similar representation properties hold with $Z$ being a 1 stable Lévy motion with drift, for certain quadratic pure jump semimartingales that have nonvanishing continuous bounded variation part related to $\pi$ (see Theorem 1).

On the other hand, if a semimartingale $X$ can be represented in the form (4) (resp. (3)), with $Z$ denoting an $\alpha$-stable (resp. a symmetric $\alpha$-stable) Lévy motion, then condition (2) holds for the jump-measure of $X$, where $\nu$ denotes the Lévy measure of $Z$.

Now recall that $\alpha$-stable Lévy motion is a quadratic pure jump semimartingale when $\alpha=1$, a purely discontinuous local martingale (in fact a martingale) when $1<\alpha<2$, a pure jump process with locally finite variation paths when $0<\alpha<1$. Thus for semimartingales $X$ in these three classes of processes (and verifying an additional assumption when $\alpha=1$ ) the results above can be globally expressed in the following representation criterion: $X$ can be represented in the form (4) (resp. (3)) with an $\alpha$-stable (resp. a symmetric $\alpha$-stable) Lévy motion $Z$ in the same class as $X$ if and only if the jump-measure of $X$ verifies condition (2) with the same $\alpha$.

Such a property can be interpreted as an analogy for the class of strictly $\alpha$-stable Lévy processes with $\alpha<2$, of the above mentioned classical result for the case $\alpha=2$, the Brownian one. From this point of view it's natural that the predictable compensator $\pi(d s, d x)$ takes place in the present case, of the angle bracket $\langle X, X\rangle$ of the continuous one.

In a forthcoming paper we will give examples of applications of the results stated here to stochastic equations. Also it should be noted that the present representation properties seem to be linked with the financial problem of option heging in incomplete markets (cf. [2]).

1. Preliminaries. Firstly we recall the basic notions and definitions. For all other notions the reader is referred to well-known treatises as e.g. [1], [6], [7], or [10].

Let $\mathbf{B}=(\Omega, \mathscr{F}, \mathbf{F}, \mathbf{P})$ be a stochastic basis satisfying «the usual conditions», i.e., $(\Omega, \mathscr{F}, \mathbf{P})$ is a complete probability space equipped with an increasing family $\mathbf{F}=\left(\mathscr{F}_{t}\right)_{t \geqslant 0}$ of sub- $\sigma$-algebras of $\mathscr{F}$; moreover, this family is right-continuous and $\mathscr{F}_{0}$ contains all P-null sets in $\mathscr{F}$.

For a process $X=\left(X_{t}\right)_{t \geqslant 0}$ defined on $(\Omega, \mathscr{F}, \mathbf{P})$, we write $(X, \mathbf{F})$ to mean that $X$ is $\mathbf{F}$-adapted. We suppose that all the processes $X$ under consideration are real-valued, satisfy $X_{0}=0$ and have all the sample paths in $\mathbf{D}=\mathbf{D}\left(\mathbf{R}_{+}, \mathbf{R}\right)$, the space of the càdlág mappings from $\mathbf{R}_{+}=[0,+\infty[$ into $\mathbf{R}$. And all the stochastic bases we consider are supposed to satisfy «the usual conditions».

Also we adopt the usual convention $0 \cdot \infty=0 / 0=0$.

Coming to stable processes and writing $\sim$ for equality in distribution, recall that an F-Lévy process $Z$ is said to be strictly $\alpha$-stable if it has the scaling (or self-similarity) property

$$
Z(s t) \sim s^{1 / \alpha} Z(t), \quad s>0, t \geqslant 0 .
$$

These assumptions characterize the law of strictly $\alpha$-stable Lévy processes (cf., for example, [4, section 4, §5]). In particular, it is known that only $0<\alpha \leqslant 2$ may occur: for $\alpha=2$, a strictly 2 -stable Lévy process $Z$ is a Wiener process (propotional to a standard Wiener process), for $\alpha<1$, it is a pure jump process with a locally finite variation and, for $1<\alpha<2$, it is a purely discontinuous martingale. Though neither property holds when $\alpha=1$, a strictly 1-stable Lévy process is still a semimartingale with vanishing continuous 
martingale component. Moreover, for any $\alpha<2$, the Lévy measure of $Z$ is of the form

$$
\nu(d x)=1_{10,+\infty[}(x) \frac{c_{+}}{x^{1+\alpha}} d x+1_{]-\infty, 0[}(x) \frac{c_{-}}{|x|^{1+\alpha}} d x
$$

with constants $c_{+} \geqslant 0, c_{-} \geqslant 0$ that are necessarily equal in the case $\alpha=1$. When $c_{+}=c_{-}=c$, the measure $\nu$ is said to be symmetric. Because of the characteristic function, in the case $\alpha \neq 1(\alpha<2) Z$ is symmetric if and only if $\nu$ is symmetric.

Set $E=\mathbf{R}-\{0\}$ and denote by $\mathscr{E}$ the Borel $\sigma$-algebra of $E$. For a process $(X, \mathbf{F})$, one defines the jump-measure as the integer valued random measure $\mu$ given on $\mathscr{B}\left(\mathbf{R}_{+}\right) \otimes \mathscr{E}$ by

$$
\mu(d t, d x)=\sum_{s>0} 1_{\left\{\Delta X_{s} \neq 0\right\}} \varepsilon_{\left(s, \Delta X_{s}\right)}(d t, d x)
$$

where $\varepsilon_{(s, x)}$ is the Dirac measure concentrated at the point $(s, x), \mathscr{B}\left(\mathbf{R}_{+}\right)$denotes the Borel $\boldsymbol{\sigma}$-algebra of $\mathbf{R}_{+}$.

When $(Z, \mathbf{F})$ is a strictly $\alpha$-stable $\mathbf{F}$-Lévy process, its jump-measure $p$ turns out to be a F-Poisson random measure (cf. [7, Chap. II, §4]) having (deterministic) F-compensator of the form

$$
q(d s, d x)=d s \otimes \nu(d x),
$$

where $\nu$ is defined in (1.1) (and is necessarily symmetric in the case $\alpha=1$ ).

In terms of stochastic integrals with respect to random measures, strictly $\alpha$-stable Lévy processes $(Z, \mathbf{F})$ can be canonically expressed as follows, $p$ denotes the jump-measure of $Z$ :

a) if $\alpha<1$, then $Z_{t}=\int_{\mathrm{j} 0, t]} \int_{E} x p(d s, d x)$;

b) if $1<\alpha<2$, then $Z_{t}=\int_{[0, t]} \int_{|x| \leqslant 1} x(p-q)(d s, d x)+\int_{j 0, t]} \int_{|x|>1} x p(d s, d x)-a t=$ $\int_{0, t]} \int_{E} x(p-q)(d s, d x)$, where $a=\left(c_{+}-c_{-}\right) /(\alpha-1)$;

c) if $\alpha=1$, then $Z_{t}=\int_{j 0, t]} \int_{|x| \leqslant 1} x(p-q)(d s, d x)+\int_{j 0, t]} \int_{|x|>1} x p(d s, d x)+a t$, where $a$ is a real number (when $a=0$ and $c>0, Z$ is called a Cauchy process (and is of course symmetric)).

2. Representation results. Let $(X, \mathbf{F})$ be a process defined on $\mathbf{B}=(\Omega, \mathscr{F}, \mathbf{F}, \mathbf{P})$ and let $\mu$ denote the jump-measure of $(X, F)$ defined by (1.2).

Let $\pi(\omega, d s, d x)$ be the F-dual predictable projection of $\mu$. We consider the following assumptions.

Assumption 1. There exist an $\mathbf{F}$-predictable process $H(s, \omega)$ and constants $c_{+} \geqslant 0$, $c_{-} \geqslant 0,0<\alpha<2$, such that

$$
\pi(\omega, d s, d x)=|H(s, \omega)|^{\alpha} q(d s, d x),
$$

where $q(d s, d x)=d s \otimes \nu(d x)$ and the measure $\nu$ is defined on $E$ by (1.1).

Assumption 2. The same as in the preceding assumption but with a symmetric $\nu$ (i.e., $c_{+}=c_{-}=c \geqslant 0$ ).

If either of these assumptions is verified with $c_{+}+c_{-}>0$, it is easy to see that $\mathbf{P}$-a.s.

$$
\int_{0}^{t}\left|H_{s}\right|^{\alpha} d s<+\infty \text { for all } t .
$$

The following theorem involves the concept of an extension of a stochastic basis for which the reader is referred to $[5$, def. 7.2, p. 89].

Theorem 1. a) Let $X$ be a quadratic pure jump F-semimartingale $X$ satisfying Assumption 2 with $\alpha=1$ and such that for all $t$

$$
X_{t}=\int_{] 0, t]} \int_{|x| \leqslant 1} x(\mu-\pi)(d s, d x)+\int_{] 0, t]} \int_{|x|>1} x \mu(d s, d x)+a A_{t},
$$


where $A_{t}(\omega)=\int_{0}^{t}\left|H_{s}(\omega)\right| d s$ and $a$ is a real number.

Then there is an extension $(\bar{\Omega}, \bar{F}, \overline{\mathbf{F}}, \overline{\mathbf{P}})=\overline{\mathbf{B}}$ of $\mathbf{B}$ on which a strictly 1-stable Lévy process $(Z, \overline{\mathrm{F}})$ with drift at and Lévy measure $\nu(d x)=1_{E}(x)\left(c / x^{2}\right) d x$ is defined such that

$$
X_{t}=\int_{10, t]}\left|H_{s}\right| d Z_{s}, \quad t \geqslant 0 .
$$

b) The assumptions are the same as in the above case a) except for the process $A$ that verifies now the relation $A_{t}(\omega)=\int_{]_{0, t}} H_{s}(\omega) d s, t \geqslant 0$.

Then there is an extension $\mathrm{B}^{*}=\left(\Omega^{*}, \mathscr{F}^{*}, \mathrm{~F}^{*}, \mathbf{P}^{*}\right)$ of $\mathbf{B}$ on which a process $\left(Z^{*}, \mathbf{F}^{*}\right)$ having the same law as $Z$ above is defined such that

$$
X_{t}=\int_{] 0, t]} H_{s} d Z_{s}^{*}, \quad t \geqslant 0 .
$$

Corollary. Let the assumptions be the same as in case a) (and in case b)) but with $a=0$ and $c>0$. Then we have both the representations

$$
X_{t}=\int_{] 0, t]}\left|H_{s}\right| d Z_{s} \quad \text { and } \quad X_{t}=\int_{] 0, t]} H_{s} d Z_{s}^{*}, \quad t \geqslant 0
$$

where $Z$ and $Z^{*}$ both are Cauchy processes defined in general on extensions of $\mathbf{B}$.

Theorem 2. a) Let $X$ be a purely discontinuous F-local martingale that verifies Assumption 1 with $\alpha$ such that $1<\alpha<2$.

Then there is an extension $\overline{\mathbf{B}}=(\bar{\Omega}, \overline{\mathscr{F}}, \overline{\mathbf{F}}, \overline{\mathbf{P}})$ of $\mathbf{B}$ on which a strictly $\alpha$-stable Lévy process $(Z, \overline{\mathrm{F}})$ with Lévy measure $\nu(d x)$ is defined such that

$$
X_{t}=\int_{j 0, t]}\left|H_{s}\right| d Z_{s}, \quad t \geqslant 0 .
$$

b) Let $(X, \mathrm{~F})$ be a process of pure jump type with locally finite variation paths. Assume that it verifies Assumption 1 with $\alpha, 0<\alpha<1$.

Then there is an extension $\overline{\mathbf{B}}=(\bar{\Omega}, \overline{\mathscr{F}}, \overline{\mathbf{F}}, \overline{\mathbf{P}})$ of $\mathbf{B}$ on which a strictly $\alpha$-stable Lévy process $(Z, \overline{\mathrm{F}})$ with Lévy measure $\nu(d x)$ is defined such that

$$
X_{t}=\int_{] 0, t]}\left|H_{s}\right| d Z_{s}, \quad t \geqslant 0 .
$$

c) Let all the assumptions in a) (resp. b)) hold. In addition we assume that $\nu$ is symmetric (so Assumption 2 holds). Then besides that given in a) (resp. b)), we have the representation

$$
X_{t}=\int_{10, t]} H_{s} d Z_{s}^{*}, \quad t \geqslant 0,
$$

where $Z^{*}$ is an $\alpha$-stable Lévy process with Lévy measure $\nu(d x)$ defined in general on an extension of $\mathrm{B}$.

$\mathrm{R}$ e $\mathrm{m}$ a $\mathrm{r} \mathbf{k}$. In a standard way the extensions of $\mathrm{B}$, which were considered in the corollary above and in point c) of the last theorem, can be chosen to be the same for $Z$ and $Z^{*}$ (cf. [9, Remark (4.1), p. 169]).

3. Proofs. Although in the present case suitable extensions of $\mathbf{B}$ can be constructed in a rather elementary manner (cf. [14]), it is a quicker way to use directly the Grigelionis representation theorem of integer-valued random measures by means of stochastic integrals with respect to Poisson ones [3].

So assume that Assumption 1 holds with $c_{+}+c_{-}>0$. Add the point $\delta=0$ to $E$ and denote by $E_{\delta}$ the locally compact space $E \cup\{\delta\}$, by $\mathscr{E}_{\delta}$ the Borel $\sigma$-algebra of $E_{\delta}$. If $B$ 
is in $\mathscr{E}$, by the change of variable $x=\left|H_{s}(\omega)\right| \cdot y$ it is simple to see that we have, for all $(\omega, s)$,

$$
1_{\left\{H_{s}(\omega) \neq 0\right\}} \cdot \int_{E} 1_{B}(x)\left|H_{s}(\omega)\right|^{\alpha} \nu(d x)=1_{\left\{H_{s}(\omega) \neq 0\right\}} \cdot \int_{E} 1_{B}\left(\left|H_{s}(\omega)\right| \cdot y\right) \nu(d y),
$$

and since $1_{B}(0)=0$,

$$
\int_{E} 1_{B}(x)\left|H_{s}(\omega)\right|^{\alpha} \nu(d x)=\int_{E} 1_{B}\left(\left|H_{s}(\omega)\right| \cdot y\right) \nu(d y) .
$$

It follows

$$
\pi(\omega, A)=\int 1_{A}\left(t,\left|H_{s}(\omega)\right| \cdot y\right) d t \nu(d y) \quad \text { if } A \in \mathscr{B}\left(\mathbf{R}_{+}\right) \otimes \mathscr{E} .
$$

Owing to this relation to the measure $\mu$ we can apply the representation theorem above quoted in the form stated in [6, Chap. XIV , 4, § b]: indeed (3.1) corresponds to Hypothesis (14.52) there with the function $h: \Omega \times \mathbf{R}_{+} \times E \longrightarrow E_{\delta}, h(\omega, t, y)=\left|H_{t}(\omega)\right| \cdot y$.

So, because of Theorem (14.56) in [6], there exists an auxiliary space $\Omega^{\prime}$ (denoted $\dot{\Omega}^{2} \times \dot{\Omega}$ in [6]) by which one can construct an extension $\overline{\mathbf{B}}$ of $\mathbf{B}$ (verifying the usual conditions)

$$
\begin{cases}\overline{\mathbf{B}}=(\bar{\Omega}, \bar{F}, \overline{\mathbf{F}}, \overline{\mathbf{P}}) & \text { with } \bar{\Omega}=\Omega \times \Omega^{\prime}, \mathbf{P}=\varphi(\overline{\mathbf{P}}) \\ \varphi: \bar{\Omega} \rightarrow \Omega & \text { being defined by } \varphi\left(\omega, \omega^{\prime}\right)=\omega,\end{cases}
$$

carrying a Poisson random measure $p\left(\left(\omega, \omega^{\prime}\right) ; \cdot\right)$ on $\mathscr{B}\left(\mathbf{R}_{+}\right) \otimes \mathscr{E}$, with the compensator $q(d t, d y)$, such that one has, for $\overline{\mathbf{P}}$-a.e. $\left(\omega, \omega^{\prime}\right)$,

$$
\mu(\omega ; A)=\int 1_{A}\left(t,\left|H_{t}(\omega)\right| y\right) p\left(\left(\omega, \omega^{\prime}\right) ; d t, d y\right) \quad \text { if } A \in \mathscr{B}\left(\mathbf{R}_{+}\right) \otimes \mathscr{E} .
$$

In other words, for $\overline{\mathbf{P}}$-a.e. $\left(\omega, \omega^{\prime}\right)$, the measure $\mu(\omega ; \cdot)$ is the restriction to $\mathbf{R}_{+} \times E$ of the image measure (on $\left(E_{\delta}, \mathscr{E}_{\delta}\right)$ ) of $p\left(\left(\omega, \omega^{\prime}\right) ; \cdot\right)$ by the function $(t, y) \longmapsto\left(t,\left|H_{t}(\omega)\right| \cdot y\right)$.

If $\nu$ is symmetric (with $c_{+}=c_{-}=c>0$ ), besides the one just given, we have another representation formula for $\mu$. Indeed, by the change of variable $x=H_{s}(\omega) \cdot y$ it is easy to see that

$$
1_{\left\{H_{s}(\omega) \neq 0\right\}} \cdot \int_{E} 1_{B}(x)\left|H_{s}(\omega)\right|^{\alpha} \nu(d x)=1_{\left\{H_{s}(\omega) \neq 0\right\}} \cdot \int_{E} 1_{B}\left(H_{s}(\omega) y\right) \nu(d y),
$$

for all $(s, \omega)$ and $B$ in $\mathscr{E}$. It follows

$$
\pi(\omega ; A)=\int 1_{A}\left(t, H_{t}(\omega) y\right) d t \nu(d y) \quad \text { if } A \in \mathscr{B}\left(\mathbf{R}_{+}\right) \otimes \mathscr{E}
$$

and thanks to the same theorem as above, using the same space $\Omega^{*}=\bar{\Omega}=\Omega \times \Omega^{\prime}$ with the same $\varphi: \bar{\Omega} \rightarrow \Omega$ as for (3.2), one can define an extension

$$
\mathbf{B}^{*}=\left(\Omega^{*}, \mathscr{F}^{*}, \mathbf{F}^{*}, \mathbf{P}^{*}\right) \quad \text { of } \mathbf{B}, \mathbf{P}=\varphi\left(\mathbf{P}^{*}\right)
$$

and a Poisson random measure $p^{*}$ on $\mathrm{B}^{*}$ with compensator $q(d t, d y)$ such that one has, for $\mathbf{P}^{*}$-a.e. $\left(\omega, \omega^{\prime}\right)$,

$$
\mu(\omega ; A)=\int 1_{A}\left(t, H_{t}(\omega) y\right) p^{*}\left(\left(\omega, \omega^{\prime}\right) ; d t, d y\right) \quad \text { if } A \in \mathscr{B}\left(\mathbf{R}_{+}\right) \otimes \mathscr{E}
$$

(see [6], loc cit). 
Now we prove Theorem 1a). If $\nu=0$, we have the trivial representation formula $X_{t}=\int_{j 0, t]}\left|H_{s}\right| d Z_{s}, t \geqslant 0$, with the degenerate $Z, Z_{t}=a t$ for all $t$.

So we assume that Assumption 2 holds with $c>0$ and $\alpha=1$. We consider the introduced above extension $\overline{\mathbf{B}}$ on which representation (3.3) holds with the Poisson random measure $p$ and set

$$
Z_{t}=\int_{] 0, t]} \int_{E} 1_{\{|x| \leqslant 1\}} x(p-q)(d s, d x)+\int_{0, t]} \int_{E} 1_{\{|x|>1\}} x p(d s, d x)+a t
$$

$(Z, \overline{\mathbf{F}})$ turns out to be a strictly 1-stable Lévy process with drift at. Since $H \circ \varphi$ is predictable on $\overline{\mathbf{B}}$, property (2.1) assures the existence of the stochastic integral of $|H \circ \varphi|$ with respect to $Z$ (see [8, Theorem 3.1(a)]). Set

$$
I_{t}=\int_{] 0, t]}\left|(H \circ \varphi)_{s}\right| d Z_{s} .
$$

Theorem 1a) will be established if we prove the following proposition.

Proposition 1. Processes $I$ and $X \circ \varphi$ are $\overline{\mathbf{P}}$-equal.

Since $\Delta I=|H| \Delta Z$ up to the $\overline{\mathbf{P}}$-equality, from (3.3) and the definition of $Z$ we have directly the following lemma.

Lemma 1. Processes $\Delta(X \circ \varphi)$ and $\Delta I$ are $\overline{\mathbf{P}}$-equal.

Then set

$$
\left\{\begin{array}{l}
N_{t}=\int_{] 0, t]} \int_{\{|x| \leqslant 1\}} x(p-q)(d s, d x), \\
K_{t}=\int_{] 0, t]} \int_{\{|x|>1\}} x p(d s, d x), \\
C_{t}=\int_{] 0, t]}\left|(H \circ \varphi)_{s}\right| d N_{s} \\
W_{t}=\int_{] 0, t]}\left|(H \circ \varphi)_{s}\right| d K_{s}
\end{array}\right.
$$

so that, for all $t, Z_{t}=N_{t}+K_{t}+a t, I_{t}=C_{t}+W_{t}+a(A \circ \varphi)_{t}$.

Moreover, if we set

$$
\begin{aligned}
M_{t} & =\int_{j 0, t]} \int_{\{|x| \leqslant 1\}} x(\mu-\pi)(d s, d x), \\
V_{t} & =\int_{j 0, t]} \int_{\{|x|>1\}} x \mu(d s, d x),
\end{aligned}
$$

by assumption we have $X_{t}=M_{t}+V_{t}+a A_{t}$, so that

$$
X \circ \varphi-I=M \circ \varphi+V \circ \varphi-W-C \text {. }
$$

Lemma 2. The process $V \circ \varphi-W$ is a purely discontinuous local martingale on $\overline{\mathbf{B}}$. $\mathrm{P}$ r o o f. Note that (3.3) is equivalent to the following property: for $\overline{\mathbf{P}}$-a.e. $\left(\omega, \omega^{\prime}\right)$

$$
\mu(\omega ; A)=\int 1_{A}\left(t,\left|H_{t}(\omega)\right| y 1_{\left\{H_{t}(\omega) \neq 0\right\}}\right) p\left(\left(\omega, \omega^{\prime}\right) ; d t, d y\right) \quad \text { if } A \in \mathscr{B}\left(\mathbf{R}_{+}\right) \otimes \mathscr{E} .
$$

Thus the pure jump process (with locally finite sets of jump times) $V \circ \varphi$ is $\overline{\mathbf{P}}$-equal to the process

$$
\int_{] 0, t]} \int_{E} 1_{\left\{|y|>\left|H_{s}(\omega)\right|^{-1}\right\}} 1_{\left\{H_{s}(\omega) \neq 0\right\}}\left|H_{s}(\omega)\right| y p\left(\left(\omega, \omega^{\prime}\right) ; d s, d y\right) .
$$


Measure $p$ being Poisson, also the process

$$
W_{t}\left(\omega, \omega^{\prime}\right)=\int_{j 0, t]} \int_{E} 1_{\{|y|>1\}} 1_{\left\{H_{s}(\omega) \neq 0\right\}}\left|H_{s}(\omega)\right| y p\left(\left(\omega, \omega^{\prime}\right) ; d s, d y\right)
$$

is of pure jump type with a locally finite number of jumps. So, writing $1_{\left\{H_{s}(\omega) \neq 0\right\}}=$ $1_{\left\{\left|H_{s}(\omega)\right|>1\right\}}+1_{\left\{0<\left|H_{s}(\omega)\right| \leqslant 1\right\}}$, we have, for all $t$

$$
\begin{aligned}
(V \circ \varphi)_{t}-W_{t}= & \int_{] 0, t]} \int_{E} 1_{\left\{\left|H_{s}\right|>1\right\}}{ }_{\left\{\left|H_{s}\right|^{-1}<|y| \leqslant 1\right\}}\left|H_{s}\right| y p(d s, d y) \\
& -\int_{] 0, t]} \int_{E} 1_{\left\{0<\left|H_{s}\right| \leqslant 1\right\}^{1}}\left\{1<|y| \leqslant\left|H_{s}\right|^{-1}\right\}\left|H_{s}\right| y p(d s, d y) .
\end{aligned}
$$

Since the pure jump process

$$
U_{t}=\int_{] 0, t]} \int_{E} 1_{\left\{0<\left|H_{s}\right| \leqslant 1\right\}} 1_{\left\{1<|y| \leqslant\left|H_{s}\right|^{-1}\right\}}\left|H_{s}\right| y p(d s, d y)
$$

has all jumps bounded by 1 , it is locally $\overline{\mathbf{P}}$-integrable and the $\overline{\mathbf{B}}$-compensator $\check{U}$ of $U$ exists and is finite. In fact, for all $t$,

$$
\begin{aligned}
\check{U}_{t} & =\int_{0}^{t} \int 1_{\left\{0<\left|H_{s}\right| \leqslant 1\right\}} 1_{\left\{1<|y| \leqslant\left|H_{s}\right|^{-1}\right\}}\left|H_{s}\right| y d s \nu(d y) \\
& =\int_{0}^{t} 1_{\left\{0<\left|H_{s}\right| \leqslant 1\right\}}\left|H_{s}\right| d s\left(\int_{E} 1_{\left\{1<|y| \leqslant\left|H_{s}\right|^{-1}\right\}} y \frac{c}{y^{2}} d y\right)=0
\end{aligned}
$$

and $U$ turns out to be a local martingale (with finite-variation paths) on $\overline{\mathbf{B}}$.

Now remark that in the last term of (3.10) the first pure jump process (with a locally finite number of jumps) is the same as the Stieltjes integral of $\left|H_{s}\right|$ with respect to the following process having paths of the same form

$$
D_{t}=\int_{j 0, t]} \int_{E} 1_{\left\{\left|H_{s}\right|>1\right\}} 1_{\left\{\left|H_{s}\right|^{-1}<|y| \leqslant 1\right\}} y p(d s, d y) .
$$

Since $D$ has all jumps bounded by 1 , it is locally $\overline{\mathbf{P}}$-integrable and the $\overline{\mathbf{B}}$-compensator $\check{D}$ of $D$ exists. As above, a simple calculation shows that $\check{D}$ is the null process so that $D$ is a $\overline{\mathrm{B}}$-local martingale. The same holds for $\int_{] 0, t]}\left|H_{s}\right| d D_{s}$ and we conclude that $V \circ \varphi-W$ is a $\overline{\mathbf{B}}$-local martingale. But $V \circ \varphi-W$ is a finite variation process, thus it is a purely discontinuous local martingale (see Lemma 4.14 b, p. 41 in [7]).

Since $M \circ \varphi$ is a purely discontinuous $\overline{\mathbf{B}}$-local martingale (see Prop. (10.46) (a) in [6]) and the same holds for $C$, combining (3.9) with Lemmas 1 and 2 we have that $X \circ \varphi-I$ is a purely discontinuous local martingale having jump process $\Delta(X \circ \varphi-I) \overline{\mathbf{P}}$-equal to zero. Using Corollary 4.19, p. 43 in [7], we conclude that $X \circ \varphi-I$ is $\overline{\mathbf{P}}$-equal to 0 and Proposition 1 is proved.

Coming to the proof of Theorem $1 \mathrm{~b}$ ), consider the introduced above extension $\mathbf{B}^{*}$ of $\mathrm{B}$ with the Poisson random measure $p^{*}$ supplying the representation formula (3.5). Then define $Z^{*}$ similarly to (3.6) but using $p^{*}$ instead of $p:\left(Z^{*}, \mathrm{~F}^{*}\right)$ is a 1-stable Lévy motion and in the same way as above, one shows that $X \circ \varphi$ is $\mathbf{P}^{*}$-equal to $\int_{j 0, \cdot]}(H \circ \varphi)_{s} d Z_{s}^{*}$.

Now the corollary is clear.

In Theorem 2a), with $c_{+}+c_{-}>0$, for all $t$ we have

$$
X_{t}=\int_{\mathrm{j} 0, t]} \int_{E} x(\mu-\pi)(d s, d x) .
$$


(In the case $\nu=0, X$ is $\mathbf{P}$-equal to the null process and, for any $H, X$ is trivially represented as $\int_{0}^{*}\left|H_{s}\right| d Z_{s}$ or $\int_{0}^{\dot{H}} H_{s} d Z_{s}$ with $Z$ the null process (degenerate case).) Then we consider an extension $\overline{\mathbf{B}}$ carrying a Poisson random measure $p$ with compensator $d t \otimes \nu(d y)$ (where $\nu$ is defined by (1.1)) that supplies the representation formula (3.3). The process $Z_{t}=\int_{j 0, t]} \int_{E} x(p-q)(d s, d x)$ is a strictly $\alpha$-stable Lévy process on $\overline{\mathbf{B}}$ and (2.3) ensures the existence of $I_{t}=\int_{j 0, t]}\left|(H \circ \varphi)_{s}\right| d Z_{s}$.

But the purely discontinuous local martingales $X \circ \varphi, I$ are $\overline{\mathbf{P}}$-equal, on account of the fact that the jump processes $\Delta(X \circ \varphi), \Delta I$ are the same, up to $\overline{\mathbf{P}}$-equality, because of (3.3).

In the case of Theorem 2c) with the assumptions referring to point a) and $c>0$, one considers an extension $\mathbf{B}^{*}$ carrying a Poisson random measure $p^{*}$ (with compensator $d t \otimes \nu(d y)$ and $c_{+}=c_{-}=c$ in (1.1)) that supplies the representation formula (3.5). As in the case of point a), one shows that the process $Z_{t}^{*}=\int_{j 0, t]} \int_{E} x\left(p^{*}-q\right)(d s, d x)$ permits the desired representation property.

The cases of Theorem $2 b$ ) and c) with the assumptions referring to b), are treated in a similar way. We omit details.

4. Final considerations. Similarly to the Brownian case, when the process $H$ in Assumptions 1 and 2 is a.s. separated from zero, there is no need to introduce the extensions $\overline{\mathbf{B}}, \mathbf{B}^{*}$ of $\mathbf{B}$ (cf. for the Brownian case [5], Theorem 7.1, p. 84 or [7], Remark 4.3, p. 173). More precisely we have the following proposition.

Proposition 2. Assume that all the assumptions of Theorem 1 (resp. Corollary, resp. Theorem 2) are in force with a nondegenerate $\nu$ (i.e., $c_{+}+c_{-}>0$ ) and the addition of the following one:

a) Process $H$ vanishes only on a $(\omega, s)$-set having $\mathbf{P}(d \omega) \otimes d s$-measure 0 .

Then all the representation properties in Theorem 1 (resp. Corollary, resp. Theorem 2) hold with processes $Z, Z^{*}$ defined on the original stochastic basis $\mathbf{B}$.

$\mathrm{P} \mathrm{r}$ o o f. Owing to the assumption a) of the proposition, in the present case using Corollary (14.63) in [6] one can directly derive representation formulas similar to (3.3), (3.5) but with respect to Poisson random measures $p, p^{*}$ on $\mathrm{B}$.

However a direct definition of $p$ is very simple now. So, when Assumption 1 holds with $c_{+}+c_{-}>0$, one defines an $\mathbf{R}_{+} \times E$-valued random measure $p$ on $\Omega$ as follows

$$
p(\omega ;[0, t] \times B)=\int_{] 0, t]} \int_{E} 1_{B}\left(\frac{x}{\left|H_{s}(\omega)\right|}\right) 1_{\left\{H_{s}(\omega) \neq 0\right\}} \mu(\omega ; d s, d x), \quad B \in \mathscr{E} .
$$

By the change of variable $y=x /\left|H_{s}(\omega)\right|$ is easy to see that

$$
1_{\left\{H_{s}(\omega) \neq 0\right\}} \int_{E} 1_{B}\left(\frac{x}{\left|H_{s}(\omega)\right|}\right)\left|H_{s}(\omega)\right|^{\alpha} \nu(d x)=1_{\left\{H_{s}(\omega) \neq 0\right\}} \int_{E} 1_{B}(y) \nu(d y), \quad B \in \mathscr{E}
$$

and to check that the B-compensator of $p$ is $q_{1}(\omega ; d s, d x)=1_{\left\{H_{s}(\omega) \neq 0\right\}} d s \otimes \nu(d x)$. Thanks to assumption a), $q_{1}$ is P-equivalent to the measure $q(d s, d x)=d s \otimes \nu(d x)$, so that $p$ is Poisson on B. Owing to Assumption 1, P-a.s. measure $\mu$ does not charge the set $\left\{s: H_{s}=0\right\} \times E$, so, for $\mathbf{P}$-a.e. $\omega$, we have

$$
\mu(\omega ; A)=\int 1_{A}\left(t,\left|H_{t}(\omega)\right| \cdot y\right) p(\omega ; d t, d y) \quad \text { if } A \in \mathscr{B}\left(\mathbf{R}_{+}\right) \otimes \mathscr{E} .
$$

When Assumption 2 holds with $c>0$, one defines $p^{*}$ on $\Omega$ as follows

$$
p^{*}(\omega ;[0, t] \times B)=\int_{] 0, t]} \int_{E} 1_{B}\left(\frac{x}{H_{s}(\omega)}\right) 1_{\left\{H_{s}(\omega) \neq 0\right\}} \mu(\omega ; d s, d x), \quad B \in \mathscr{E} .
$$


Also this measure is seen to be Poisson on $\mathrm{B}$ with the compensator $d s \otimes \nu(d x)$ and for $\mathbf{P}$-a.e. $\omega$, we obtain the representation formula

$$
\mu(\omega ; A)=\int 1_{A}\left(t, H_{t}(\omega) \cdot y\right) p^{*}(\omega ; d t, d y) \quad \text { if } A \in \mathscr{B}\left(\mathbf{R}_{+}\right) \otimes \mathscr{E} .
$$

Using (4.1) or, according to the different cases, (4.2), all the above representation properties are obtained on $\mathbf{B}$ with the same proofs as above.

Finally, suppose that a semimartingale $X$ is given on a stochastic basis $\mathbf{B}$ and that there exists on $\mathbf{B}$ a predictable process $H$ and an $\alpha$-stable (resp. a symmetric $\alpha$-stable) Lévy motion $Z(0<\alpha<2)$ such that

$$
X_{t}=\int_{\mathrm{j} 0, t]}\left|H_{s}\right| d Z_{s} \quad\left(\text { resp. } X_{t}=\int_{\mathrm{j} 0, t]} H_{s} d Z_{s}\right) .
$$

Then the jump measures $\mu, p$ of $X, Z$ respectively, verify (4.1) (resp. the analogous of (4.2) with $p$ in place of $p^{*}$ ) and by the change of variable $x=\left|H_{s}(\omega)\right| \cdot y\left(\operatorname{resp.} x=H_{s}(\omega) \cdot y\right)$ is easy to see that the compensator $\pi$ of $\mu$ has the form in Assumption 1 (resp. Assumption 2) with respect to the Lévy measure $\nu$ of $Z$.

Combining last remark with the above results and still denoting by $\pi$ the compensator of the jump-measure $\mu$ of a generic semimartingale, we have the following criterion.

Representation criterion. In the class of semimartingales $X$ which can be decomposed as in Theorem 1 a) with a predictable $H$ and $a \in \mathbf{R}$ (resp. which are purely discontinuous local martingales, resp. which are pure jump processes with locally finite variation paths) Assumption 2 with $\alpha=1$ and the same $H$ if $a \neq 0$ (resp. Assumption 1 with $\alpha, 1<\alpha<2$, resp. Assumption 1 with $\alpha, 0<\alpha<1$ ) for $\pi$, is sufficient (as explained in $\S 2$ ) and necessary in order that the representation

$$
X_{t}=\int_{] 0, t]}\left|H_{s}\right| d Z_{s} \quad t \geqslant 0
$$

may hold, $Z$ being an $\alpha$-stable Lévy motion.

A similar property holds for the representation

$$
X_{t}=\int_{] 0, t]} H_{s} d Z_{s}, \quad t \geqslant 0
$$

with a symmetric (for all $\alpha, 0<\alpha<2$ ) $Z, X$ being decomposed, when $\alpha=1$, as in Theorem $1 \mathrm{~b}$ ).

$\mathrm{R}$ e $\mathrm{m}$ a $\mathrm{r}$ k. In order to prove Theorem (2.5) of [13], in the particular case considered there, we have already established a representation formula of the same form as that in Theorem $2 \mathrm{c}$ ) here, with the process $Z^{*}$ defined on the original stochastic basis $\mathbf{B}$. In that case indeed, Assumption a) of Proposition 2 is verified by the process corresponding to $H_{s}(\omega)$ which is, in the notation of [13], $b\left(X_{s}-(\omega)\right)$ : see Lemma $(2.20)$ there. For the representation formula, cf. Lemmas (2.23) and (2.26) there.

\section{REFERENCES}

1. Dellacherie C., Meyer P. A. Probabilités et Potentiel. Chap. V à VIII. Paris: Hermann, 1980.

2. Föllmer H., Schweizer M. Hedging of contingent claims under incomplete information. - In: Applied Stochastic Analysis, Stochastic Monographs. Vol. 5, Gordon \& Breach, 1991, p. 389-414.

7 Теория вероятностей и ее применения, № 4 
3. Grigelionis $B$. On the representation of integer-valued random measures by means of stochastic integrals with respect to the Poisson measure. - Lit. Math. J., v. 11, № 1, p. 93-108, 1971.

4. Itô $K$. Lectures on Stochastic Processes. Tata Institute of Fund. Research. Berlin: Springer-Verlag, 1984.

5. Ikeda N., Watanabe S. Stochastic Differential Equations and Diffusion Processes. Amsterdam: North-Holland, 1981.

6. Jacod J. Calcul Stochastique et Problèmes de Martingales. Lecture Notes in Math., Vol. 714, Berlin; Springer, 1979.

7. Jacod J., Shiryaev A. N. Limit Theorems for Stochastic Processes. Berlin: Springer, 1987.

8. Kallenberg $O$. Some time change representations of stable integrals, via predictable transformations of local martingales. - Stochastic Process. Appl., 1992, v. 40, p. 199223.

9. Karatzas I., Shreve S. E. Brownian Motion and Stochastic Calculus, 2nd ed. New York: Springer, 1994.

10. Liptser R. Sh., Shiryaev A. N. Theory of Martingales. Dordrecht: Kluwer, 1989.

11. Protter Ph. Stochastic Integration and Differential Equations. A New Approach. Berlin: Springer. 1990.

12. Rosiński J., Woyczyński $W$. A. On Itô stochastic integration with respect to $p$-stable motion: inner clock, integrability of sample paths, double and multiple integrals. Ann. Probab., 1986, v. 14, p. 271-286.

13. Zanzotto $P$. A. On solutions of one-dimensional stochastic differential equations driven by stable Lévy motion. - Stochastic Process. Appl., 1997, v. 68, p. 209-228.

14. Zanzotto $P$. A. Representation of Quadratic Pure Jump Semimartingales as Stable Integrals. Technical Report 2.282.1061, Dip. di Matematica, Univ. di Pisa, July, 1997. 